\title{
Vulval lymphangiectasia: 2 case reports
}

\section{Gunjan Gulati $^{1 *}$, Rohit Bhagat ${ }^{2}$, Tripta S. Bhagat ${ }^{3}$}

\begin{abstract}
${ }^{1}$ Department of Obstetrics and Gynecology, VMMC and Safdarjung Hospital, New Delhi, India ${ }^{2}$ Department of Internal Medicine, Guru Teg Bahadur Hospital, New Delhi, India

${ }^{3}$ Department of Surgery, Santosh Medical College and Hospital, Ghaziabad, Uttar Pradesh, India
\end{abstract}

Received: 02 July 2017

Accepted: 29 July 2017

\author{
*Correspondence: \\ Dr. Gunjan Gulati, \\ E-mail: gunjan7565@gmail.com
}

Copyright: (c) the author(s), publisher and licensee Medip Academy. This is an open-access article distributed under the terms of the Creative Commons Attribution Non-Commercial License, which permits unrestricted non-commercial use, distribution, and reproduction in any medium, provided the original work is properly cited.

\begin{abstract}
Vulval lymphangiectasia is a rare cutaneous condition of lymphatic channels in deep dermal and subcutaneous layers. It can be congenital condition or might develop secondary to tuberculosis, surgery, radiotherapy, crohn's disease etc. Very few cases have been reported in the literature so far. Diagnosis is mainly clinical aided with histopathology. Various treatment modalities are available for this condition. Surgical treatment is the most commonly used method to treat vulvar lymphangiectasia and has offered promising results. In this report, we present 2 cases of vulval lymphangiectasia- one congenital case and other acquired after tuberculosis.
\end{abstract}

Keywords: Acquired, Congenital, Tuberculosis, Vulval lymphangiectasia

\section{INTRODUCTION}

Lymphangiectasia is a condition resulting from damage to the lymphatic channels leading to impaired lymph drainage and its collection leading to lymphedema. ${ }^{1}$

It is seen at various sites like vulva, intestine and conjunctiva. These can be congenital or acquired. Acquired cutaneous lymphangiectasia (ACL), also called as acquired lymphangioma, is a rare condition in which dilated lymphatic channels arise following damage to previously normal deep lymphatics, whereas lymphangioma circumscriptum (LC) is used when lymphatic channel dilation occurs because of congenital malformations of the lymphatic system involving the skin and the subcutaneous tissues. ${ }^{1}$

The literature review with pubmed revealed 13 cases of congenital and 78 cases of acquired vulval lymphangiectasia. We describe 2 cases of vulval lymphangiectasia- 1 congenital and 1 acquired after tuberculosis.

\section{CASE REPORT}

\section{Case 1}

A 23 year old woman came to the outpatient department with complaints of raised lesions over vulva since 5 years.

She received a course of antitubercular treatment for 6 months following pulmonary tuberculosis at 5 years of age. She had 3 normal vaginal deliveries and her menstrual cycles were regular. On Local examination multiple small $<1 \mathrm{~cm}$ nodular lesions were seen over right labia majora (Figure 1).

A biopsy was taken under local anaesthesia and histopathology revealed dilated thin walled lymphatic channels consistent with vulval lymphangiectasia. 
Available therapeutic options were offered to the patient but she refused treatment.

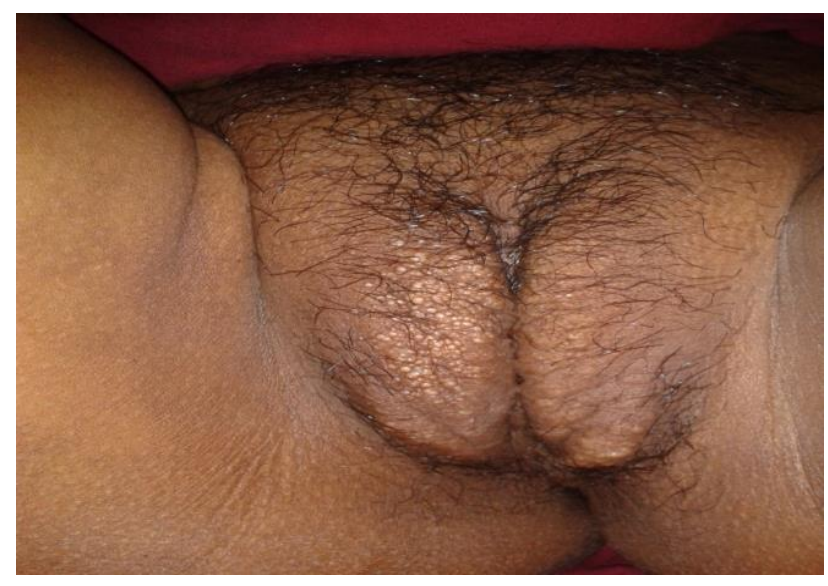

Figure 1: Small nodules over right labia majora.

\section{Case 2}

A 17 year old girl reported with itching over vulva worse during menstruation since 4 years. She attained menarche at 13 years of age and her menstrual cycles were regular with no other complaints. There was no history consistent with the predisposing factors such as tuberculosis, crohn's disease etc. The patient came for visit on second day of her menses and local examination revealed translucent vesicles with very minimal clear, watery, nonfoul smelling discharge distributed over both labia majora and more near the perineum (Figure 2). A biopsy was taken under local anaesthesia and histopathology revealed dilated lymphatics lined by normal endothelium. No treatment could be offered as patient was lost to follow up.

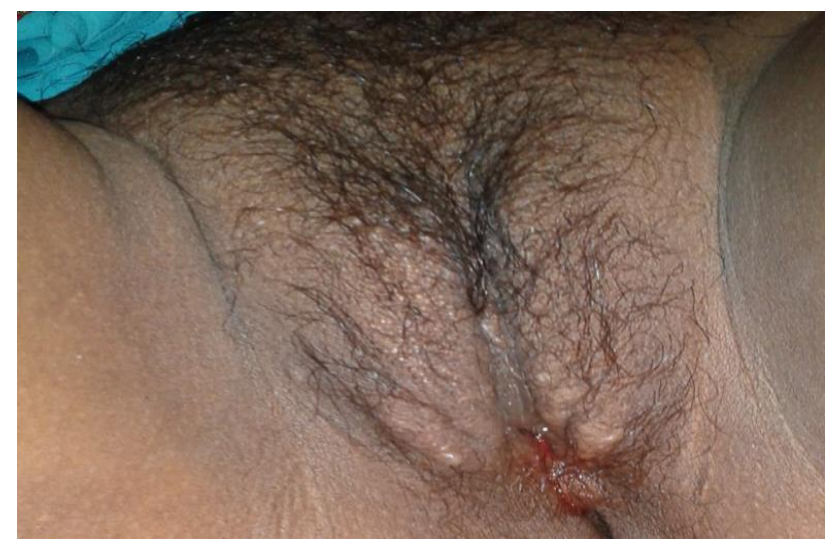

Figure 2: Small translucent vesicles over bilateral labia majora.

\section{DISCUSSION}

Vulval lyphangiectasia is a rare condition resulting from obstruction of the lymphatic channels resulting in dilatation of the lymph channels. Only few cases are reported in the literature. It can be acquired or congenital.
Acquired lymphangiectasia occur after conditions such as tuberculosis, filariasis, Crohn's disease, surgery, trauma, radiation, malignancy and genital mutilation which may cause damage to the lymphatic channels resulting in lymph accumulation in dermal lymphatics leading to vesicle formation. ${ }^{1-3}$

There is no difference between congenital and acquired lymphangiectasia on clinical and histopathological grounds, however congenital present early in childhood without any predisposing factors and acquired occur later in life and are usually preceded by some risk factor. Although there is no difference in the treatment options available for both congenital and acquired cases.

The exact pathogenesis of lymphangiectasia is still not well understood. The vesicles associated with lymphangiectasia are suggested to represent saccular dilatations of local superficial lymphatics. These vesicles develop secondary to increased intralymphatic pressure as a result of accumulation of lymph in the superficial vessels caused by damage to previously normal deep lymphatics thereby causing lymphedema. ${ }^{4}$

The clinical presentation of vulval lymphangiectasia is diverse. It may present as vesicles, fleshy nodules, hyperkeratotic lesions or as warts..$^{2,3,5,6}$ The most common presentation is fluid oozing out of the vesicles. Rarely patient may present as cellulitis or it may develop in later stages as a complication of vulval lymphangiectasia. Awareness about this rare entity can help in suspecting the condition and help in formulating a diagnosis and aid in treatment. Diagnosis is usually based on clinical examination supported with biopsy for histopathology which shows hyperkeratosis and acanthosis of the epidermis with multiple dilated lymphatics in the papillary dermis. Rarity of the condition may commonly lead to misdiagnosis as warts, molluscum or herpes. Other differential diagnosis include mucin secreting metastatic adenocarcinoma mimicking acquired lymphangioma, benign lymphangioendothelioma and syringoma. ${ }^{1}$

Treatment must aim at prevention of secondary infection and development of cellulitis. Treatment options include laser therapy, sclerotherapy, cryotherapy and surgical excision. Surgical excision with vulval reconstruction has shown good results. ${ }^{7}$ There is no standard treatment for this condition and the treatment modality offered must focus on good cosmetic result, prevention of recurrence, protection from infections and patient's choice. Recently Winters et al described the technique of Lymphovenous anastomosis and secondary resection for the management of vulval lymphangiectasia. ${ }^{8}$

Reccurence of the condition and keloid formation are two major complications encountered in these patients post treatment. Keloid formation is especially common after laser therapy. Malignant potential has been reported in the form of lymphangiosarcoma and squamous cell 
carcinoma in patients with vulval lymphangiectasia, thus follow up is essential to detect and treat recurrence in early stages.

\section{CONCLUSION}

Vulval lymphangiectasia is a rare cutaneous disorder with only few cases reported in the literature. Knowledge about this rare entity may raise suspicion of the condition and help formulating a diagnosis thus helping the clinician to provide treatment accordingly.

Funding: No funding sources Conflict of interest: None declared

Ethical approval: The study was approved by the Institutional Ethics Committee

\section{REFERENCES}

1. Chang MH, Shiao GH, Tseng CR. Lymphangiectasia - Report of one case and review of the literature. Dermatol Sinica. 1997;15:275-9.

2. Singh N, Kumari R, Thappa DM. Vulval lymphangiectasia secondary to tubercular lymphadenitis. Indian $\mathbf{J}$ Sex Transm Dis. 2007;28:38-9.
3. Haneef NS, Ramachandra S, Metta AK, Haritha K. Lymphangiectasias of vulva. Indian Dermatol Online J. 2011;2:40-2.

4. Gulati G, Das B, Deepika. Vulval lymphangiectasia secondary to tuberculosis: a case report and review of literature. Sch J Med Case Rep. 2014;2(9):629-31.

5. Amouri M, Masmoudi A, Boudaya S, Amouri A, Ben Ali I, Bouassida $S$ et al. Acquired lymphangioma circumscriptum of the vulva. Dermatol Online J. 2007; 13:10.

6. Horn LC, Kuhndel K, Pawlowitsch T, Leo C, Einenkel J. Acquired lymphangioma circumscriptum of the vulva mimicking genital warts. Eur J Obstet Gynecol Reprod Biol. 2005;123:118-20.

7. Okur MI, Köse R, Yildirim AM, Cobanoğlu B. Lymphangiectasia of the vulva accompanying congenital lymphedema. Dermatol Online J. 2009;15:13.

8. Winters H, Tielmans HJ, Ulrich DJ. Lymphovenous anastomosis and secondary resection for noonan syndrome with vulvar lymphangiectasia. Plast Reconstr Surg Glob Open. 2016;4(8):e1007.

Cite this article as: Gulati G, Bhagat $\mathrm{R}$, Bhagat TS. Vulval lymphangiectasia: 2 case reports. Int J Reprod Contracept Obstet Gynecol 2017;6:4158-60. 